\title{
Phase transitions in dissipative Josephson chains
}

\author{
P. A. Bobbert, R. Fazio, ${ }^{*}$ and Gerd Schön \\ Department of Applied Physics, Delft University of Technology, 2628 CJ Delft, The Netherlands \\ G. T. Zimanyi \\ Department of Physics, University of California, Davis, Davis, California 95616
}

(Received 25 September 1989)

\begin{abstract}
We study the zero-temperature phase transitions of a chain of Josephson junctions, taking into account the quantum fluctuations due to the charging energy and the effects of an Ohmic dissipation. We map the problem onto a generalized Coulomb gas model, which then is transformed into a sine-Gordon field theory. Apart from the expected dipole unbinding transition, which describes a transition between globally superconducting and resistive behavior, we find a quadrupole unbinding transition at a critical strength of the dissipation. This transition separates two superconducting states characterized by different local properties.
\end{abstract}

\section{INTRODUCTION}

In recent years interest in the properties of mesoscopic tunnel junctions, junction arrays, and granular superconductors has been growing (see, e.g., the articles in Ref. 1). With modern lithographic techniques it is possible to fabricate regular arrays of Josephson junctions that show a transition between a globally superconducting and a resistive state. In two-dimensional classical arrays this transition is of the Kosterlitz-Thouless-Berezinskii (KTB) type, ${ }^{2}$ and occurs at a critical temperature $T_{\mathrm{KTB}}$ below the superconducting transition temperature of the individual islands. It has been recognized ${ }^{3}$ that in these systems quantum effects induced by the charging energy of the metallic islands can play an important role. When the geometrical capacitance of the islands is of the order of picofarad the Coulomb energy $E_{C}=e^{2} / 2 C$ may exceed the Josephson energy $E_{J}$. In this case the quantum fluctuations lower the coherent transition temperature $T_{\mathrm{KTB}}$, and, below a critical value of the ratio $E_{J} / E_{C}$, global phase coherence is prevented even at $T=0$. The coherent transition has been investigated by means of several analytical ${ }^{4,5}$ and numerical ${ }^{6}$ methods. These approaches cannot be applied directly to the case of chains of Josephson junctions because of the difference between the relevant topological excitations in these lowdimensional systems. Bradley and Doniach ${ }^{7}$ mapped the quantum Josephson chain on a $(1+1)$-dimensional (one space and one time dimension) classical $X Y$ model. The inverse temperature $\hbar \beta$ and the charging energy of the quantum chain play the role of the system size in time direction and of the temperature of the classical problem, respectively. The coherent transition in this case is again of the KTB type.

A series of experiments, both on granular superconductors ${ }^{8}$ and arrays of Josephson junctions ${ }^{9}$ showed an additional interesting feature: The low-temperature properties of the network depend strongly on the normal sheet resistance $R$ of the system. Only below a critical value of $R$ of the order of $h / 4 e^{2} \approx 6.5 \mathrm{k} \Omega$, the system reaches a globally superconducting state (zero resistance of the whole array). The experiments suggest that this is a sample independent, universal value. The theoretical work devoted to study the transition starts from the lattice version of the Caldeira-Leggett model ${ }^{10}$ for Ohmic dissipation, or from the action obtained by Ambegaokar, Eckern, and Schön ${ }^{11}$ which describes the effect of quasiparticle tunneling. The model actions have been studied by variational calculations, ${ }^{12}$ in coarse graining approaches, ${ }^{13}$ and by other means. ${ }^{14}$ The dissipation tends to suppress the quantum fluctuations and gives rise to the observed transition. But there is no indication from these studies that the value of the critical normal sheet resistance should be universal, although its dependence on sample parameters may be weak.

Also a single junction shows a transition as a function of dissipation. ${ }^{15}$ Thus, one can expect that local quantum fluctuations affect the coherent properties of the array as well. Indeed an instanton analysis ${ }^{16}$ in the large $E_{J} / E_{C}$ regime indicated the possibility of a further phase for weak dissipation governed by strong local quantum fluctuations. The question of what the properties of this phase are, and, in particular, whether the array is in a resistive or a globally superconducting state, has not yet been settled. Very recently the dissipative Josephson chain has been studied in more detail in Refs. 17 and 18. However, we feel that the problem has not yet been solved satisfactorily. For instance, the conclusions reached in Refs. 17 and 18 do not agree completely.

It is the purpose of this paper to work out in more detail the properties of the phase transitions in a chain of Josephson junctions. We assume that the junctions are shunted by Ohmic resistors $R_{S}$ and that the quasiparticle contribution can be disregarded. We map the action of the Josephson chain on the statistical mechanics problem of a charged gas in a $(1+1)$-dimensional space-time dual lattice by means of the Villain transform ${ }^{19}$ in the limit of large $E_{J} / E_{C}$ and by means of an expansion in $E_{J}$ in the opposite limit. The dissipation induces a highly anisotropic interaction between the topological excitations 
(vortices) of the system. The properties of the gas model are analyzed further by considering the related sineGordon field theory. ${ }^{20}$ In particular, the location of the phase transition is determined by the lines in the phase diagram at which the sine-Gordon field changes from massive to massless. In the ordered phase of the dissipative quantum chain, where the vortices are bound in dipoles, one cannot disregard the residual interaction between the dipoles (in contrast to the case of the isotropic $X Y$ model or the nondissipative quantum chain). This becomes important at a critical strength of the dissipation and introduces a second phase transition characterized by a binding of dipoles into quadrupoles. This transition has been discussed in different terms in Ref. 16. Our formulation provides an understanding of this transition and allows us to draw conclusions about the response and fluctuations of the chain in the different phases.

\section{THE MODEL}

The model from which we will start our analysis is defined by the following action (putting $\hbar=1$ ):

$$
\begin{aligned}
& A[\vartheta]= \sum_{i} \int_{0}^{\beta} d \tau\left[\frac{1}{16 E_{C}}\left[\frac{d \vartheta_{i}}{d \tau}\right]^{2}-E_{J} \cos \nabla_{x} \vartheta_{i}(\tau)\right] \\
&+\frac{1}{2} \sum_{i} \int_{0}^{\beta} d \tau \int_{0}^{\beta} d \tau^{\prime} \alpha\left(\tau-\tau^{\prime}\right) \\
& \times\left[\nabla_{x} \vartheta_{i}(\tau)-\nabla_{x} \vartheta_{i}\left(\tau^{\prime}\right)\right]^{2}
\end{aligned}
$$

The three terms in the action defined are the charging energy, the Josephson coupling, and the Ohmic dissipative term, respectively. ${ }^{21}$ The label $i$ denotes the position of the island in the chain. We have defined

$$
\nabla_{x} \vartheta_{i}(\tau)=\vartheta_{i+1}(\tau)-\vartheta_{i}(\tau)
$$

as the lattice derivative in the spatial direction, and $\vartheta$ is the phase of the superconducting order parameter. The time Fourier transform of the dissipative kernel is

$$
\alpha(\omega)=-\frac{\alpha}{4 \pi}|\omega|, \quad \alpha=\frac{h}{4 e^{2} R_{S}} .
$$

The associated partition function

$$
Z=\int \prod_{i} D \vartheta_{i}(\tau) \exp (-A[\vartheta])
$$

is specified once we fix the boundary condition for the allowed paths $\vartheta_{i}(\tau)$. Here the proper choice is $\vartheta_{i}(0)=\vartheta_{i}(\beta)$ because, in the presence of Ohmic dissipation, which changes the charge in a continuous fashion, the states at 0 and $2 \pi$ are distinguishable. ${ }^{22}$ As is clear from Eq. (2), we consider here the case in which only the diagonal elements of the capacitance matrix are nonzero (the so-called self-charging model). The other extreme case, in which only the nearest-neighbor capacitances are considered, is trivial, since the system decouples into independent junctions. ${ }^{12}$ As in the standard theory of the KTB transition, we will map the problem defined above into an equivalent statistical mechanical problem of interacting charges.

\section{THE VILLAIN TRANSFORMATION}

We first consider the limit $E_{J} \gtrsim 8 E_{C}$. In this case, a straightforward way to perform this transformation to the interacting gas of charges is to use the Villain transformation. ${ }^{17,19}$ It amounts to replacing the Josephson nonlinear cosine term by a "periodic Gaussian" potential. This requires that we consider a lattice in time direction with lattice spacing $\Delta \tau$ and introduce a set of integers $n(\mathbf{r})$ at each of the $N$ discrete space-time lattice points $\mathbf{r}=(i, \tau)$. The resulting partition function is

$$
\begin{aligned}
Z=\sum_{\{n(\mathbf{r})\}} \int D \vartheta(k, \omega) \exp \left\{-\frac{1}{2} N^{-1} \sum_{k, \omega}[\right. & \left|\frac{\omega^{2}}{8 E_{C}}+\frac{\alpha}{\pi}\right| \omega|(1-\cos k)||\vartheta(k, \omega)|^{2} \\
& \left.\left.+E_{J}|[1-\exp (i k)] \vartheta(k, \omega)-2 \pi n(k, \omega)|^{2}\right]\right\} .
\end{aligned}
$$

In this form we used the strong-coupling expansion for the effective Villain coupling constant. Its use requires that the lattice spacing in the time direction $\Delta \tau$ should not be taken too small. On the other hand, for the lattice version of the path integral $\Delta \tau$ has to be small. Both conditions can be satisfied simultaneously only if $E_{J} / 8 E_{C}>1$. One can extend the method by using the weak-coupling form of the Villain transformation. ${ }^{23}$ We do not make much use of this extension since we will use a different approximation in the limit of small $E_{J} / E_{C}$.

It is easy to perform in (5) the integration over the spin-wave part of the partition function. This yields

$$
Z_{\mathrm{SW}}=\prod_{k, \omega} \pi^{1 / 2}\left[\frac{\omega^{2}}{8 E_{C}}+\frac{\alpha}{\pi}|\omega|(1-\cos k)+2 E_{J}(1-\cos k)\right]^{-1 / 2}
$$

Completing the square in (5) we arrive at

$$
Z=Z_{\mathrm{SW}} \sum_{\{p(\mathbf{r})\}} \exp \left[-\frac{1}{2} N^{-1} \sum_{k, \omega} 2 \pi p(k, \omega) G_{0}(k, \omega) 2 \pi p(-k,-\omega)\right],
$$


where the second factor is the partition function of a lattice gas of vortices $p(r)$. The interaction (in the longwavelength limit) is given by

$$
G_{0}(k, \omega)=\frac{J+(\alpha / 2 \pi) k^{2} /|\omega|}{\omega^{2}+k^{2}+(\alpha / 2 \pi) J^{-1} k^{2}|\omega|} .
$$

Here we defined the coupling constant

$$
J=\left(E_{J} / 8 E_{C}\right)^{1 / 2}
$$

and we scaled the frequency $\omega$ in units of $\left(8 E_{C} E_{J}\right)^{1 / 2}$. The new field

$$
p(\mathbf{r})=\nabla_{\tau} n(\mathbf{r})=n(i, \tau+\Delta \tau)-n(i, \tau)
$$

is also an integer field since the derivative is defined on the lattice. The singularity at $k=\omega=0$ implies that the gas of vortices is neutral. In order to illustrate the properties of the gas let us consider the strong-coupling limit $J \gg 1$. In this case the interaction (8) between the topological charges can be approximated by

$$
G_{0}(k, \omega) \approx \frac{J}{k^{2}+\omega^{2}}+\frac{\alpha}{2 \pi|\omega|} .
$$

This means it consists of an isotropic part, as in the absence of dissipation, plus a strongly anisotropic attraction in time direction between vortices located on the same space lattice point. As it will become clear later the anisotropic part of the interaction introduces new qualitative features in the phase diagram.

\section{THE SINE-GORDON MODEL AND THE PHASE DIAGRAM}

When deriving quantitative results for the phase diagram it is convenient to map the gas model defined in (7) onto an equivalent sine-Gordon field theory. This mapping is discussed in the literature,$^{20,24}$ so we omit the derivation here. As in Ref. 25, we introduce a new parameter, the activity $y_{0}$, which controls the strength of the fluctuations of the topological charges. The grand partition function of this generalized model can be expressed in terms of the field theory in a new variable $\varphi(\mathbf{r})$ defined in the continuous space

$$
\begin{aligned}
Z\left(y_{0}\right) & =Z_{\mathrm{SW}} \int D \varphi(\mathbf{r}) \exp \left(-H_{\mathrm{sG}}\right), \\
H_{\mathrm{sG}}= & \frac{1}{2} \int d^{2} r d^{2} r^{\prime} \varphi(\mathbf{r}) G_{0}^{-1}\left(\mathbf{r}-\mathbf{r}^{\prime}\right) \varphi\left(\mathbf{r}^{\prime}\right) \\
& +2 y_{0} \int d^{2} r \cos [2 \pi \varphi(\mathbf{r})],
\end{aligned}
$$

where $G_{0}$ is given by Eq. (8).

The KTB transition is characterized by the unbinding of vortex-antivortex pairs and, according to Minnhagen, ${ }^{20}$ is located at the points in the phase diagram where the mass of the sine-Gordon field vanishes. The calculation of the field mass has to be performed self-consistently. We consider the propagator

$$
G\left(\mathbf{r}-\mathbf{r}^{\prime}\right)=\left\langle\varphi(\mathbf{r}) \varphi\left(\mathbf{r}^{\prime}\right)\right\rangle_{s G}
$$

and make the following ansatz with $\mathbf{q}=(k, \omega)$ :

$$
G^{-1}(q)=G_{0}^{-1}(q)+\kappa .
$$

The mass $\kappa$ is related to the self-energy by the selfconsistency relation

$$
\kappa=-\Sigma(\mathbf{q}=0, \kappa) \text {. }
$$

The ansatz (15) for the propagator is sufficient only in the disordered phase, where $\kappa$ describing the screening of free vortices, is nonzero. The evaluation of the self-energy can be performed in a perturbative expansion in the activity $y_{0}$. In Eqs. (14)-(16) we did not introduce the longitudinal dielectric constant. The reason is that we will work out the results in first order in the activity only and at this level the dielectric constant is equal to one. In second order, in the perturbation expansion, we would find corrections to the transition point, but these are only of a quantitative nature. In the perturbation theory of the sine-Gordon model it is possible to perform a resummation of a class of diagrams (tadpoles), and this leads to a renormalization of the activity. Thus, all these diagrams can be disregarded provided one considers an expansion around the renormalized activity (vertex).

In first order in $y_{0}$ the self-energy does not depend on the wave vector. Hence,

$$
\Sigma_{1}(\mathrm{q}, \kappa)=-8 \pi^{2} y_{0} \exp \left[-2 \pi^{2} G_{0}(\mathbf{r}=0, \kappa)\right] .
$$

The phase boundary in the parameter space is found from (16) and (17) in the limit $\kappa \rightarrow 0$. The technical steps are presented in the Appendix. We obtain the following result:

$G(\mathbf{r}=0, \kappa) \approx-\left(\frac{1}{4 \pi} J+\frac{1}{2 \pi^{2}} \alpha\right) \ln (\kappa)$ for $\kappa \rightarrow 0$.

Substituting this expression into Eq. (16) we obtain the self-consistency equation for $\kappa$

$$
\kappa=8 \pi^{2} y_{0} \kappa^{\pi J / 2+\alpha} \text {. }
$$

The relation (19) has nontrivial solutions for $\kappa$ in the regime of small $J$ and $\alpha$, which we identify as the disordered phase. The quantity $\kappa$ in this phase has the meaning of a screening length. As the coupling constants increase, the solution for $\kappa$ approaches zero. This locus of point defines a phase transition line in the $J-\alpha$ diagram (for $y_{0} \rightarrow 0$ )

$$
1-\frac{\pi}{2} J-\alpha=0
$$

Above the transition line (20) the system is in an ordered state; the charges are bound in dipoles. In the disordered phase below the line (20) the charges are free. In the absence of Ohmic dissipation, the result (20) reproduces that of Bradley and Doniach. ${ }^{7}$ In the small- $\alpha$ limit it coincides with a perturbative result of Guinea. ${ }^{26}$ For stronger dissipation the transition line moves towards small $J$. In this regime, but for not too small $J$, we should use the weak-coupling form of the Villain transformation. ${ }^{19,23}$ The first correction is $J_{\text {eff }} \approx J(1-1 / J)$; this implies that the transition line is not straight but bends upward, favoring the disordered state. Finally, for very small $J$, the Villain transformation ceases to be sufficient. However, in this limit we can pursue a different approach 
which we will discuss in Sec. VI. Anticipating the result, that in the limit $J \rightarrow 0$ there is a fixed point at $\alpha=1$, we see that the transition line eventually has to become steeper again to connect to this fixed point. Before we consider the small- $J$ limit we will discuss a remarkable property of the ordered phase in the presence of the anisotropic interaction (8).

\section{THE QUADRUPOLE UNBINDING TRANSITION}

A key hypothesis in the standard treatment of the KTB transition is that once vortices are bound in dipoles the residual interaction between dipoles can be disregarded because it does not induce further critical behavior in the system. In our case, however, due to the strong anisotropy of the interaction, this hypothesis is not valid anymore. Further analysis is carried out most easily in the limit $J \gg 1$. In this case the vortices form a dilute gas of tightly bound pairs and it is safe to assume that their size is of the order of one lattice spacing. For the configurations shown in Figs. 1(a) and 1(b) for large distances the interaction (12) between dipoles reduces to

$$
\begin{aligned}
E_{d d}= & J f\left(\mathbf{d}_{1}, \mathbf{d}_{2}, \mathbf{r}_{d d}\right) \frac{1}{r_{d d}^{2}} \\
& +\frac{\alpha}{\pi^{2}} \ln \tau_{d d}\left[\delta\left(x_{d d}\right)+\frac{1}{2} \delta\left(\left|x_{d d}\right|-1\right)\right] .
\end{aligned}
$$

Here $\mathbf{r}_{d d}=\left(x_{d d}, \tau_{d d}\right)$ refers to the dipole-dipole distance and $f\left(\mathbf{d}_{1}, \mathbf{d}_{2}, \mathbf{r}_{d d}\right)$ is a function of the relative dipoledipole orientation. Apart from the first term, which describes a screened interaction as in the case of the isotropic $X Y$ model, the dipoles interact via a logarithmic interaction in time direction. This interaction is not screened and leads to additional features. For the configuration shown in Fig. 1(c), the interaction is fully screened.

The important configurations of dipoles are depicted in

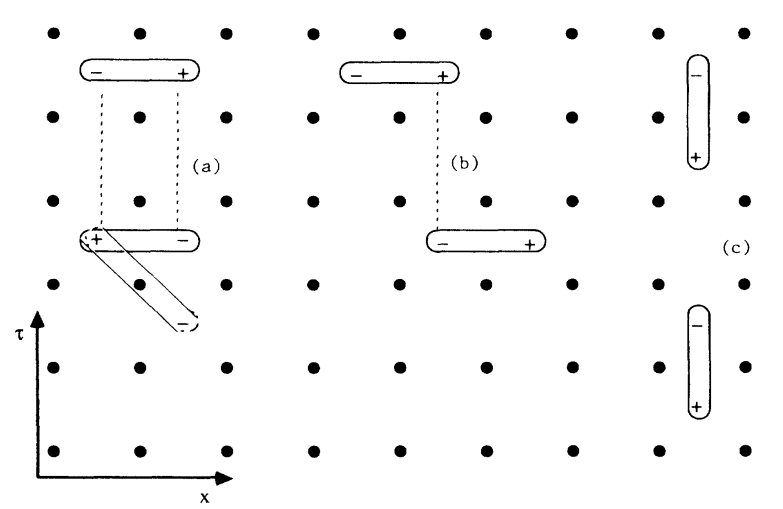

FIG. 1. We display in the space-time lattice the binding of vortices into dipoles and the interaction between dipoles. Configuration (a) shows two dipoles at the same space lattice points. Their binding into a quadrupole is responsible for the transition at $\alpha=\frac{1}{2}$. The configuration (b) is not stable and will rotate towards the configuration (c) which is fully screened. The ratio between the dipole length and the dipole-dipole separation has to be considered much greater than one.
Fig. 1(a). They form a one-dimensional (dipole) gas with a logarithmic interaction. In order to describe the properties of this gas we proceed along the same lines as outlined above for the two-dimensional vortex problem. We do not repeat the derivation but we notice that the coupling strength of the logarithmic dipole-dipole interaction is twice that of the corresponding vortex-antivortex interaction. Finally we arrive at the self-consistency relation

$$
\kappa=\text { const } \kappa^{2 \alpha}
$$

This means, we find an additional phase transition at a critical line

$$
\alpha=\frac{1}{2} \text { for } J \gg 1
$$

For $\alpha<\frac{1}{2}$ the dipoles are free to move in the time direction, for $\alpha>\frac{1}{2}$ the dipoles are bound in a gas of quadrupoles.

Turning to the configurations of the type drawn in Fig. 1(b) we notice that they can be disregarded. The reason is that these configurations are not stable, since the first part of the interaction (21) produces a nonzero torque and tends to rotate them into the orientation depicted in Fig. 1(c). In this orientation the interaction is fully screened. Furthermore, these configurations become unimportant in the limit of vanishing activity due to the charge neutrality in the $\tau$ direction. Extending the present approach one can consider more complex clusters. However, it is easy to see that the interaction between larger clusters is always screened so that one can stop the analysis at the quadrupole unbinding transition (23).

\section{THE REGION $E_{J} \ll E_{C}$}

As already stressed, the approach developed in the previous sections is reliable only in the region $J>1$. But also in the opposite limit we can use a (different) duality transformation to express the problem in terms of a gas of interacting "charges." In order to do this we extend Schmid's approach ${ }^{15}$ to a chain of Josephson junctions and represent the Josephson term of the action (1) by

$$
\begin{aligned}
\exp \left(E_{J} \sum_{\mathbf{r}} \cos \nabla_{x} \vartheta(\mathbf{r})\right] \\
=\sum_{n=0}^{\infty} \frac{\left(E_{J} / 2\right)^{n}}{n !} \sum_{\{s(\mathbf{r})\}} \exp \left[i \sum_{\mathbf{r}} s(\mathbf{r}) \nabla_{x} \vartheta(\mathbf{r})\right] .
\end{aligned}
$$

Again we introduced a set of integer charges $s(\mathbf{r})$ at the space-time lattice sites $r$, but they differ from the charges $n(\mathbf{r})$ used in the Villain transformation. Using Eq. (24) we rewrite the partition function as 


$$
Z=\int d^{N} \vartheta(\mathbf{r}) \sum_{n=0}^{\infty} \frac{\left(E_{J} / 2\right)^{n}}{n !} \sum_{\{s(\mathbf{r})\}} \exp \left[i \sum_{\mathbf{r}} s(\mathbf{r}) \nabla_{x} \vartheta(\mathbf{r})-A_{C}[\vartheta]-A_{D}[\vartheta]\right),
$$

where $A_{C}$ and $A_{D}$ are the charging and dissipative part of the action (1). The Gaussian integrations can be performed which yields a spin wave [differing from (6) by the absence of the $E_{J}$ term] and a "charge" part

$$
Z=Z_{\mathrm{SW}}\left(E_{J}=0\right) \sum_{n=0}^{\infty} \frac{\left(E_{J} / 2\right)^{n}}{n !} \sum_{\{s(\mathbf{r})\}} \exp \left[-\frac{1}{2} N^{-1} \sum_{k, \omega} s(k, \omega) D_{0}(k, \omega) s^{*}(k, \omega)\right]
$$

with the new propagator (in the long-wavelength limit)

$$
D_{0}(k, \omega)=\frac{k^{2}}{\frac{\omega^{2}}{8 E_{C}}+\frac{\alpha}{2 \pi}|\omega| k^{2}} .
$$

The singularity of the propagator at $\omega=0$ implies that, at each space point, the gas is neutral along the time direction. The interaction depends again logarithmically on $\tau$

$$
\begin{aligned}
D_{0}(x, \tau)= & -\frac{2}{\alpha} \delta_{x=0} \ln \frac{\tau}{\tau_{0}} \\
& +\frac{16}{\pi} E_{C} \gamma^{2}\left[\frac{1}{x}-\frac{\gamma}{\sqrt{2 \pi \tau}} f\left(\frac{\gamma x}{\sqrt{2 \pi \tau}}\right)\right],
\end{aligned}
$$

where the auxiliary function $f(u)$ is expressed in terms of Fresnel integrals

$$
f(u)=\left[\frac{1}{2}-C(u) \cos ^{2}\left(\frac{\pi}{2} u\right)+\left[\frac{1}{2}-S(u)\right] \sin ^{2}\left(\frac{\pi}{2} u\right)\right.
$$

and the constant $\gamma$ is $\gamma=\left(2 \pi / \alpha 8 E_{C}\right)^{1 / 2}$. When studying (28) it is useful to define a "diffusion cone" with the boundary $x_{\mathrm{dc}}=\gamma^{-1}(8 \pi \tau)^{1 / 2}$. Then (28) reduces to

$$
D_{0}(x, \tau)=-\frac{2}{\alpha} \delta_{x=0} \ln \frac{\tau}{\tau_{0}}+\frac{16}{\pi} E_{C} \gamma^{2} \frac{1}{x}
$$

if $x$ and $\tau$ are inside the diffusion cone $x \leq x_{\mathrm{dc}}$, and

$$
D_{0}(x, \tau)=-\frac{2}{\alpha} \delta_{x=0} \ln \frac{\tau}{\tau_{0}}+\text { const } \frac{\tau^{2}}{x^{5}}
$$

otherwise. Although the interaction is not critical in the spatial direction it involves many islands so that it requires some care to investigate the properties of the phase transition.

As in the last sections, we map the model (26) into a sine-Gordon field theory in order to locate the phase transition. We do not repeat the derivation, but point out the differences: (i) the factor $2 \pi$ contained in the cosine term of Eq. (14) is missing now, (ii) the Josephson energy $E_{J}$ plays the role of the activity of the gas obtained from the Villain transformation. With the definition

$$
D^{-1}(k, \omega, \kappa)=D_{0}^{-1}(k, \omega)+\kappa,
$$

the self-consistency equation (to first order in $E_{J}$ ) reads

$$
\kappa=2 E_{J} \exp \left[-\frac{1}{2} D(x=0, \tau=0, \kappa)\right]
$$

The evaluation of $D(x=0, \tau=0, \kappa)$ gives (in the limit of small $\kappa$ )

$$
D(x=0, \tau=0, \kappa) \approx-\frac{2}{\alpha} \ln \kappa+\text { const } .
$$

The transition point is then readily obtained

$$
\alpha=1 \text { for } E_{J} \rightarrow 0 \text {. }
$$

This result agrees fully with the renormalization-group approach of Chakravarty et al. ${ }^{16}$ Although the transition (35) occurs at the same value as the single junction transition ${ }^{15}$ two differences must be stressed. First, in the present case, the phase of an island and not the phase difference across a junction is localized for $\alpha \geq 1$. Second, although the interaction (28) reduces to an independent island description as far as the critical properties are concerned, it does not reduce to that in general. The response and other physical properties of the system can depend on the spatial structure.

\section{RESULTS AND DISCUSSION}

We summarize our analysis of a chain of Josephson junctions shunted by normal resistors in the phase diagram shown in Fig. 2. The solid lines show our results obtained from the different approaches described in Secs.

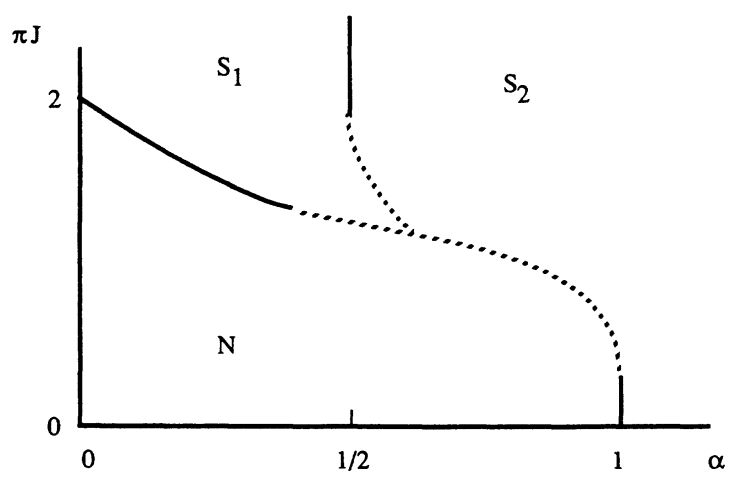

FIG. 2. We show the $T=0$ phase diagram of the dissipative Josephson chain in the $J-\alpha$ plane. The $N$ phase refers to the normal state; $S_{1}$ and $S_{2}$ are both superconducting, in $S_{1}$ the dipoles are bound in quadrupoles. 
IV-VI, i.e., Eqs. (20), (23), and (35). They are connected by dotted lines which represent our present understanding but which need further justification. We distinguish three regions $N, S_{1}$, and $S_{2}$.

The phase boundary defined by Eq. (20) (with corrections from the weak-coupling form of the Villain transformation) separates the resistive $(N)$ and the globally superconducting states $\left(S_{1}\right.$ and $\left.S_{2}\right)$.

The phase transition defined by (23) between $S_{1}$ and $S_{2}$ resembles the one described in Ref. 16. It emerges naturally from the properties of the topological excitations of the system, and our approach provides a transparent interpretation of this transition. In both regions $S_{1}$ and $S_{2}$ the vortices form dipoles. A dipole oriented in the space direction describes a phase slip by $2 \pi$ of a single island of the chain (see Fig. 3). This implies that the phase slips of two neighboring junctions have opposite sign, so that there is no voltage drop across the sample. In contrast the isolated charges, present in $N$, lead to a voltage drop across the chain. The phase changes corresponding to the quadrupole configuration shown in Fig. 1(a) are shown in Fig. 3. It describes forward and backward phase slips of one island at different times. This means that in $S_{2}$, even with a local probe at a single junction, a vanishing average voltage is seen. In contrast, in $S_{1}$, a local probe observes a finite voltage. ${ }^{27}$ At the quadrupole unbinding transition $S_{1}-S_{2}$ the time correlations of phase-slip processes change and the properties of the voltage fluctuations will change accordingly. Thus, the transition can be observed by studying the correlation functions of the system.

In the limit of small $J$, after expansion in the Josephson coupling, we also arrived at the picture of a charged gas. It has a transition at $\alpha=1$ [see Eq. (35)], consistent with renormalization-group treatments. ${ }^{16}$ Although this transition point coincides with the transition of a single junction, we expect that the properties of the ordered and disordered phases depend on properties of the array.

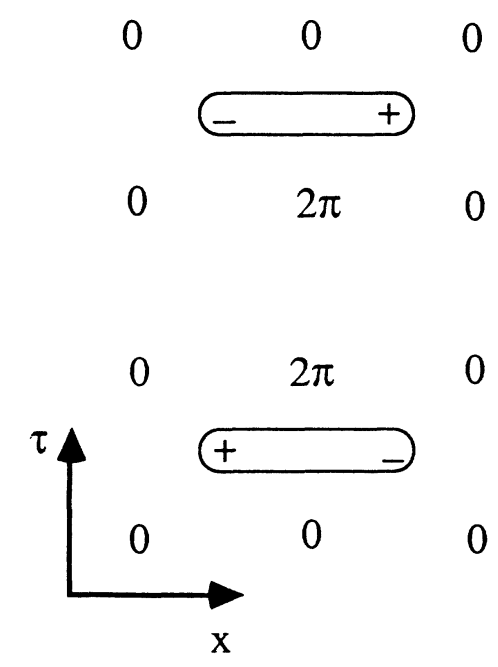

FIG. 3. We illustrate the time evolution of the phases of the islands in the presence of the topological excitations in the system corresponding to the configuration of Fig. 1(a).
The charges $s(\mathbf{r})$, which we introduced in the small- $J$ limit, are related to real electric charges [more precisely, at the space-time site $\mathbf{r}$ where $s(\mathbf{r})=1$, a Cooper pair is tunneling across a junction]. They are different from the topological charges $p(\mathbf{r})$ (the vortices) of the large- $J$ description. Nevertheless, we notice that a naive extrapolation of the result (20), which is based on the vortex picture, predicts the same transition point as (35), which is based on the charges $s(r)$. Furthermore, in the limit $J=0$ at $\alpha=1$ the two descriptions in terms of vortices and in terms of the charges $s(\mathbf{r})$ are self-dual, suggesting again that there exists a phase transition at this point. However, since the Villain transformation and the vortex picture obtained from it are not valid in this limit we cannot draw definite conclusions from this coincidence.

Zwerger ${ }^{17}$ used the vortex gas picture, obtained from the Villain transformation, down to $J=0$ and derives from it a transition at $\alpha=1$. He further speculates about the properties of the phase at $J \approx 0, \alpha>1$, suggesting that it has local phase coherence but, e.g., no Meissner effect. And he suggests that there exists a further phase transition at $\alpha>1$ and small nonzero $J$ between this phase and the phase with global order (for large $J$ ). But, as discussed above, the Villain transformation fails at small $J$, which casts doubt on conclusions derived from it. We agree that the transition at $J \approx 0, \alpha=1$ is of a different nature than the vortex-binding transition in the large- $J$ limit. However, it is also possible that there is a continuous crossover in the nature of the transition between the resistive and the superconducting states with increasing $J$, without a further phase transition. Such a picture emerged from the variational calculations. ${ }^{12}$ In any case, we point out that both our approach and the renormalization-group work $^{16}$ yield only one transition for $J \approx 0$. More conclusive results could be obtained from a study of the response functions, e.g., by extending the approach of Eckern and Pelzer ${ }^{28}$ to include the spatial dependence contained in (26).

Our phase diagram Fig. 2 is similar to, but less structured than the one obtained by Korshunov. ${ }^{18,29}$ He considers a model that is different from ours - apart from the self-capacitance, he includes a nearest-neighbor (NN) capacitance. This has an important consequence, it allows him to extend - for large-NN capacitance - the Villain transformation down to small $J$. It appears that the NN capacitance does not change the structure if his description. Therefore, some of his conclusions may also have implications for our model. We find the same transition point at $\alpha=0$. At nonzero small $\alpha$, we predict a tilted transition line, whereas Korshunov's line is independent of $\alpha$. He obtains his result in second order in the activity of the charges, and then takes the limit of vanishing activity. We can mention that independent calculations of Guinea ${ }^{26}$ and the general trend observed in all approaches support a tilted line. We find the same $S_{1}-S_{2}$ transition at large $J$, but we provide a physical interpretation. We agree that the nature of the normal-tosuperconducting transition changes with decreasing $J$. This is a direct consequence of the $S_{1}-S_{2}$ transition, which also implies that the $N-S$ transition changes its character. The dielectric functions of the dipole-ordered 
and the quadrupole-ordered phases will be different. Therefore we can understand a kink at the triple point. Korshunov observes a change in the effective dimensionality at this point. Finally, Korshunov finds a phase with "local phase coherence" for small $J$ and $\alpha>1$, similar to Zwerger. However, we repeat that in the model with large-NN capacitance, the Villain transformation is valid in this regime, while it does not apply in the model with self-capacitance only. We, therefore, cannot judge the implications of this result for our model.

\section{ACKNOWLEDGMENTS}

We acknowledge many stimulating discussions with $\mathbf{S}$. Chakravarty, G. Falci, U. Geigenmüller, G. Giaquinta, F. Guinea, M. L. Horbach, H. M. Jaeger, and A. Muramatsu. We also acknowledge the support of the Fondaziona A. della Riccia (R.F.), the Koninklijke Nederlandse Akademie van Wetenschappen (P.A.B.), and the research program of the Stichting voor Fundamenteel Onderzoek der Materie (FOM) (G.S.).

\section{APPENDIX}

In this appendix we present, in more detail, the steps involved in the evaluation of the Green's function with mass $\kappa$. After the Fourier transformation we have

$$
G_{0}(\mathbf{r}=0, \kappa)=\int \frac{d k}{2 \pi} \frac{d \omega}{2 \pi} G_{0}(k, \omega, \kappa)=J \int \frac{d k}{2 \pi} \frac{d \omega}{2 \pi} \frac{1}{\left(k^{2}\right) /\left(1+\lambda k^{2} /|\omega|\right)+\omega^{2}+\kappa},
$$

where $\lambda=(\alpha / 2 \pi) J^{-1}$ and the integrations extend over the first Brillouin zone $-\pi \leq k, \omega \leq \pi$. We split the integral into a sum of two integrals

$$
\begin{aligned}
G_{0}(\mathbf{r}=0, \kappa) & =\int \frac{d k}{2 \pi} \frac{d \omega}{2 \pi} \frac{J}{k^{2}+\omega^{2}+\lambda|\omega| k^{2}+\kappa\left(1+\lambda k^{2} /|\omega|\right)}\left(1+\lambda \frac{\kappa^{2}}{|\omega|}\right) \\
& =A(\kappa)+\lambda B(\kappa) .
\end{aligned}
$$

Both integrals in Eq. (A2) diverge in the limit $\kappa \rightarrow 0$. It can be easily proven that the divergent part of $A(\kappa)$ is unchanged if the terms proportional to $\lambda$ in the denominator of the integrand are dropped. The integration is then straightforward, which yields

$$
A(\kappa)=-\frac{1}{4 \pi} J \ln (\kappa) \text {. }
$$

In the second integral in Eq. (A2) the integration over $k$ can be performed leading to

$$
B(\kappa)=\int_{-\pi}^{\pi} \frac{d \omega}{2 \pi} \frac{1}{\omega+\lambda \kappa+\lambda \omega^{2}}-\frac{1}{\pi} \int_{-\pi}^{\pi} \frac{d \omega}{2 \pi} \frac{\left(\kappa|\omega|+|\omega|^{3}\right)^{1 / 2}}{\left(\lambda \kappa+|\omega|+\lambda \omega^{2}\right)^{3 / 2}} \arctan \left(\frac{\lambda \kappa+|\omega|+\lambda \omega^{2}}{\kappa|\omega|+|\omega|^{3}}\right)^{1 / 2} .
$$

The second integral in the expression is convergent in the limit $\kappa \rightarrow 0$, and in the same limit we can drop the term $\lambda \omega^{2}$ in the first integral. The final result is

$$
B(\kappa)=-\frac{1}{\pi} J \ln (\kappa)
$$

from which we obtain Eq. (19).

*Permanent address: Istituto di Fisica, Facoltà di Ingegneria, viale A. Doria 6, 95129, Catania, Italy.

${ }^{1}$ Proceedings of the NATO Advanced Research Workshop on Coherence in Superconducting Networks, edited by J. E. Mooij and G. Schön [Physica B 152 (1988)].

${ }^{2}$ J. M. Kosterlitz and D. J. Thouless, J. Phys. C 6, 1181 (1973); V. L. Berezinskii, Zh. Eksp. Teor. Fiz. 59, 907 (1970) [Sov. Phys._JETP 32, 493 (1970)].

${ }^{3}$ B. Abeles, Phys. Rev. B 15, 2828 (1977).

${ }^{4}$ E. Simanek, Phys. Rev. B 22, 495 (1980); K. B. Efetov, Zh. Eksp. Teor. Fiz. 78, 2017 (1980) [Sov. Phys._JETP 51, 1015 (1980)].

${ }^{5}$ P. Fazekas, B. Mühlschlegel, and M. Schröter, Z. Phys. B 57, 193 (1984); R. Fazio and G. Giaquinta, Phys. Rev. B 34, 4909 (1986); R. S. Fishman, Phys. Rev. Lett. 53, 89 (1989).
${ }^{6}$ L. Jacobs, J. V. José, and M. A. Novotny, Phys. Rev. Lett. 53, 2177 (1984).

${ }^{7}$ R. M. Bradley and S. Doniach, Phys. Rev. B 30, 1138 (1984).

${ }^{8}$ H. M. Jaeger et al., Phys. Rev. B 34, 4920 (1986); 40, 182 (1989).

${ }^{9}$ L. G. Geerligs and J. E. Mooij, Physica B 152, 212 (1988); L. J. Geerligs, M. Peters, L. E. M. de Groot, A. Verbruggen, and J. E. Mooij, Phys. Rev. Lett. 63, 326 (1989).

${ }^{10}$ A. O. Caldeira and A. J. Leggett, Ann. Phys. (N.Y.) 149, 374 (1983).

${ }^{11}$ V. Ambegaokar, U. Eckern, and G. Schön, Phys. Rev. Lett. 48, 1745 (1982); U. Eckern, V. Ambegaokar, and G. Schön, Phys. Rev. B 30, 6419 (1984).

${ }^{12}$ S. Chakravarty, G. L. Ingold, S. Kivelson, and A. Luther, Phys. Rev. Lett. 56, 2303 (1986); A. Kampf and G. Schön, 
Phys. Rev. B 36, 3651 (1987); M. P. A. Fischer, ibid. 36, 1917 (1987).

${ }^{13}$ S. V. Panyukov and A. D. Zaikin, Phys. Lett. A 124, 325 (1987); S. Chakravarty, S. Kivelson, G. T. Zimanyi, and B. I. Halperin, Phys. Rev. B 37, 7256 (1988); A. Kampf and G. Schön, ibid. 37, 5954 (1988); Physica B 152, 239 (1988); W. Zwerger, J. Low Temp. Phys. 72, 291 (1988).

${ }^{14}$ U. Eckern and A. Schmid, Phys. Rev. B 39, 6441 (1989); J. Choi and J. V. José, Phys. Rev. Lett. 62, 1904 (1989); R. Fazio, G. Falci, and G. Giaquinta, Solid State Commun. 71, 275 (1989); B. Mirhashem and R. A. Ferrell, Physica C 152, 361 (1988); Phys. Rev. B 37, 649 (1988).

${ }^{15}$ A. Schmid, Phys. Rev. Lett. 51, 1506 (1983).

${ }^{16}$ S. V. Paniukov and A. D. Zaikin, J. Low Temp. Phys. 75, 361 (1989); S. Chakravarty, G. L. Ingold, S. Kivelson, and G. T. Zimanyi, Phys. Rev. B 37, 3283 (1988).

${ }^{17}$ W. Zwerger, Europhys. Lett. 9, 421 (1989).

${ }^{18}$ S. E. Korshunov, Europhys. Lett. 9, 107 (1989).

${ }^{19}$ J. Villain, J. Phys. 36, 581 (1975); M. P. A. Fischer and D. H. Lee, Phys. Rev. B 39, 2756 (1989).
${ }^{20}$ P. Minnhagen, Rev. Mod. Phys. 59, 1001 (1987); and (unpublished).

${ }^{21}$ For the case in which also fluctuations in the modulus are considered see, G. Falci, R. Fazio, and G. Giaquinta, Phys. Rev. B 39, 8984 (1989).

${ }^{22}$ G. Schön, and A. D. Zaikin, Physica B 152, 203 (1988).

${ }^{23}$ In the low-coupling regime the effective Villain coupling constant is replaced by $\left(E_{J} \Delta \tau\right)_{\mathrm{eff}}=-2\left[\ln \left(2 / E_{J} \Delta \tau\right)\right]^{-1}$.

${ }^{24}$ S. Samuel, Phys. Rev. D 18, 1916 (1978).

${ }^{25}$ J. V. José, L. P. Kadanoff, S. Kirkpartick, and D. R. Nelson, Phys. Rev. B 16, 1217 (1977).

${ }^{26} \mathrm{~F}$. Guinea (private communication).

${ }^{27}$ Such an interpretation of the transition was suggested earlier by A. D. Zaikin (private communication). This conjecture is confirmed by our analysis.

${ }^{28}$ U. Eckern and F. Pelzer, Europhys. Lett. 3, 131 (1987).

${ }^{29}$ After submission of this paper an extended version of Ref. 18 became available. S. E. Korshunov, Zh. Eksp. Teor. Fiz. 95, 1058 (1989) [Sov. Phys._JETP 68, 609 (1989)]. 